\title{
Treating Melanoma in Situ During a Pandemic with Telemedicine and a Combination of Imiquimod, 5-Fluorouracil, and Tretinoin
}

\author{
William J. Nahm (D) - Eran C. Gwillim • Evangelos V. Badiavas • \\ Anna J. Nichols • Robert S. Kirsner · Laurence H. Boggeln • \\ John T. Shen
}

Received: October 24, 2020 / Published online: January 17, 2021

(C) The Author(s) 2021

\section{ABSTRACT}

The recent coronavirus disease 2019 (COVID19) pandemic has created a quandary for the physician in terms of evaluating and treating cutaneous skin cancers, particularly melanomas. At the onset of the pandemic, many planned medical and surgical visits for skin

W. J. Nahm ( $\square)$

New York University Grossman School of Medicine, New York, NY, USA

e-mail: william.nahm@nyulangone.org

E. C. Gwillim · E. V. Badiavas · A. J. Nichols

R. S. Kirsner

Dr. Phillip Frost Department of Dermatology and

Cutaneous Surgery, University of Miami Miller

School of Medicine, Miami, FL, USA

\section{A. J. Nichols · R. S. Kirsner}

Sylvester Comprehensive Cancer Center, Miami, FL, USA

L. H. Boggeln

Department of Family Medicine, Western University

School of Medicine, Pomona, CA, USA

L. H. Boggeln

Department of Family Medicine, UHS Southern

California Medical Education Consortium,

Temecula, CA, USA

J. T. Shen

Shen Dermatology, Temecula, CA, USA cancers were postponed. Physicians and patients have had to balance the risk of exposure to severe acute respiratory syndrome coronavirus 2 (SARS-CoV-2) with that of worsening morbidity and mortality due to delays in skin cancer treatments. We present a male patient who had two melanoma-in-situs (MISs) that were treated during the COVID-19 pandemic with a combination of topical imiquimod 5\% cream, 5 -fluorouracil $2 \%$ solution, and tretinoin $0.1 \%$ cream. The successful treatments occurred without in-person visits and with the aid of telemedicine. Although surgery is the standard for the treatment of melanoma in situ, this case demonstrates an effective viable treatment modality for MIS during a pandemic situation.

Keywords: COVID-19;

Fluorouracil; Imiquimod; Melanoma-in-situ; SARS-CoV-2; Skin cancer; Telemedicine; Topical therapy; Tretinoin 


\section{Key Summary Points}

The COVID-19 pandemic has created a delay in access to treatment of cutaneous skin cancers, including melanomas.

Two melanoma-in-situs (MISs) were treated with a topical combination of imiquimod 5\% cream, 5-fluorouracil 2\% solution, and tretinoin $0.1 \%$ cream (IMI/ 5-FU/TRET) without in-person office visits and with a store-and-forward telemedicine application.

The use of IMI/5-FU/TRET with telemedicine is a useful option to manage and treat MIS remotely during the time of a pandemic.

\section{DIGITAL FEATURES}

This article is published with digital features to facilitate understanding of the article. You can access the digital features on the article's associated Figshare page. To view digital features for this article go to https://doi.org/10.6084/m9. figshare.13293041.

\section{INTRODUCTION}

During the coronavirus disease of 2019 (COVID19) pandemic, the National Comprehensive Cancer Network (NCCN), numerous medical organizations, and physicians have proposed delaying localized treatments for skin cancers, including melanomas $[1,2]$. Physicians, who are conditioned to treating cutaneous cancers expeditiously, had to weigh the benefits of early cancer treatment for their patients versus potentially exposing them to severe acute respiratory syndrome coronavirus 2 (SARS-CoV-2) $[3,4]$. The pandemic also ushered in the widespread adoption and acceptance of using telemedicine modalities for medical care by physicians and patients [5]. This shift in healthcare delivery has allowed patients to practice social distancing and avoid in-person visits, but there are many limitations in terms of medical care, especially with skin cancer treatments [6-8]. Although melanoma-in-situ (MIS) is typically treated by surgical excision, reports have shown that topical imiquimod, 5-fluorouracil, and certain retinoids used as a mono or dual therapy have been effective treatments for melanoma and MIS [9-14]. Here we present the case of a patient who had two effective treatments of MISs in a pandemic setting with a combination of topical imiquimod 5\% cream, 5-fluorouracil $2 \%$ solution, and tretinoin $0.1 \%$ cream. During the treatment phase, the patient had no personto-person medical visits and was monitored remotely with a telemedicine app.

\section{CASE REPORT}

A 66-year-old male physician had two biopsyproven MISs on the left forearm (1.2-cm irregular pigmented patch) (Figs. 1a, 2a, b) and left upper arm (6-mm asymmetric brown-colored macule) diagnosed before the COVID-19 pandemic. He reported being a non-smoker but had a history of asthma with occasional bouts of acute bronchitis. His medications included theophylline and an albuterol inhaler. The patient (Fitzpatrick skin type I) also had an extensive sun-exposure history as a child and presented with multiple keratinocyte carcinomas (KCs) and actinic keratoses in adulthood. In the past, he had successful treatments of his KCs with the triple combination approach of topical imiquimod 5\% cream, 5-fluorouracil 2\% solution, and tretinoin $0.1 \%$ cream.

After his diagnosis of two MISs, the patient was instructed to see a surgical oncologist for the removal of both lesions. Due to the onset of the pandemic, which created a lack of available surgical appointments, he could not address both his MISs and had to resort to telemedicine. With the reality of these restrictions, unwillingness to have visits that could risk SARS-CoV2 exposure, and concerns about having melanomas, the patient requested an alternative treatment that required no in-person interaction. After extensive consideration of the higher 

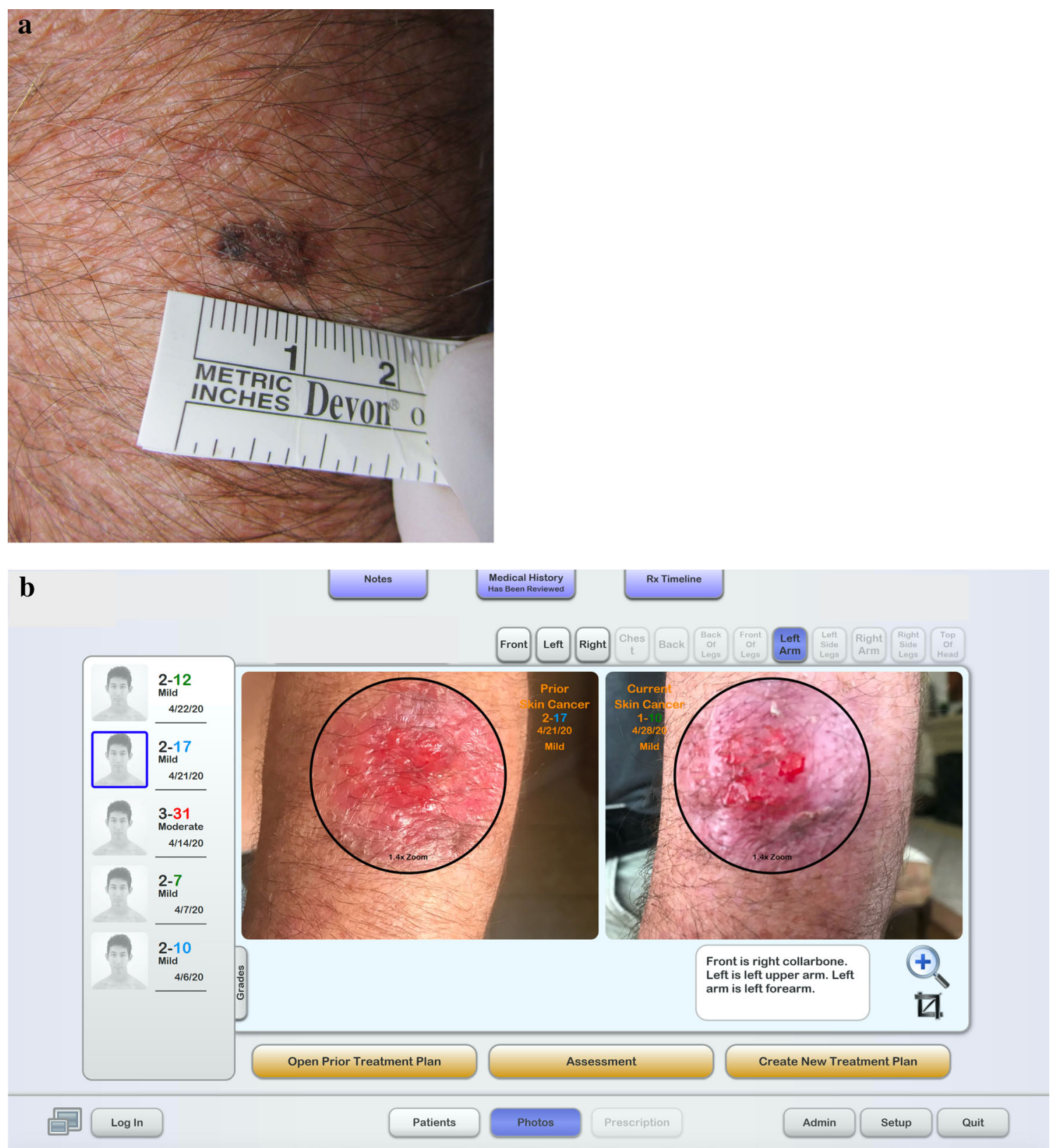

Fig. 1 a Clinical image of melanoma in situ on the left forearm. b Dashboard of the store-and-forward app (DermTRAC) demonstrating a side bar with numerical scoring of patient-reported treatment-related adverse events $(0$, none; 1 , minimal; 2 , mild; 3 , moderate; 4 , severe) and sum total in colored numbers of scores based

risks of undergoing non-surgical therapies and being fully informed of all options, the patient opted for a topical therapy protocol. The upon patient-answered questions of adverse event. There is also a date of visit journal submission under each score. The right side of the screen shows transformed images of treated melanoma in situ on the left forearm. In the sequential images during treatment, there is a demonstration of erythema, oozing, and ulceration

treatment involved a combination of $1 / 5$ packet of imiquimod $5 \%$ cream, one drop of 5 -fluorouracil $2 \%$ solution, and $1 / 5$ of a pea-sized 

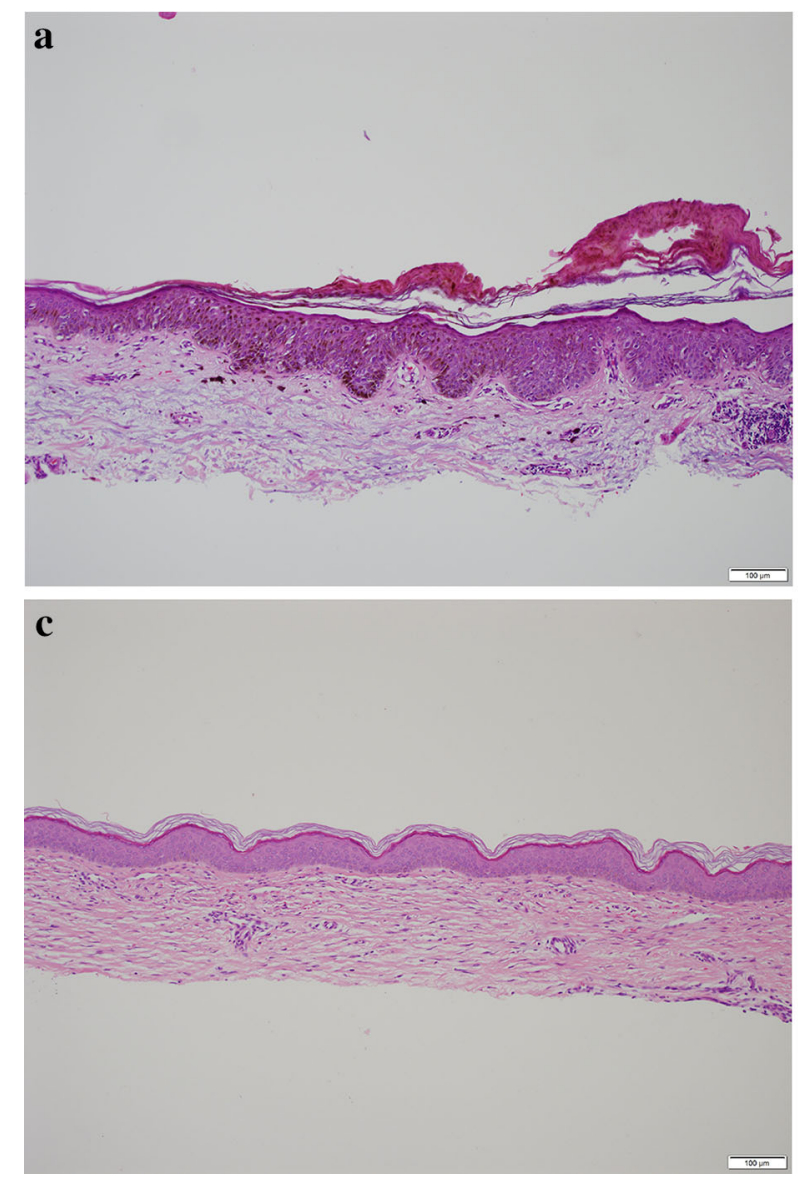

Fig. 2 a Hematoxylin and eosin staining of melanoma in situ of the left forearm. There is a mildly thickened epidermis with atypical melanocytes at all levels of the epidermis organized in a pagetoid pattern. Single cells have an expanded cytoplasm with a dusty tan pigment and enlarged nuclei. Some of the nuclei have a single nucleolus.

quantity of tretinoin $0.1 \%$ cream applied to each of his MISs with a bandage overnight. The patient was instructed to use 30 applications within a 42-day period, but he completed his treatment protocols over 30 days. During these treatments, the patient employed a store-andforward app (DermTRAC; Winchester, CA, USA), which allowed him to report his progress and the side effects of the treatment (Fig. 1b) as well as receive customized medical instructions. Although the patient had a moderate amount of oozing, erythema, crusting, scaling, and burning in both treatment areas (Fig. 1b), he was able to tolerate the cutaneous side effects and used the topical medications in compliance

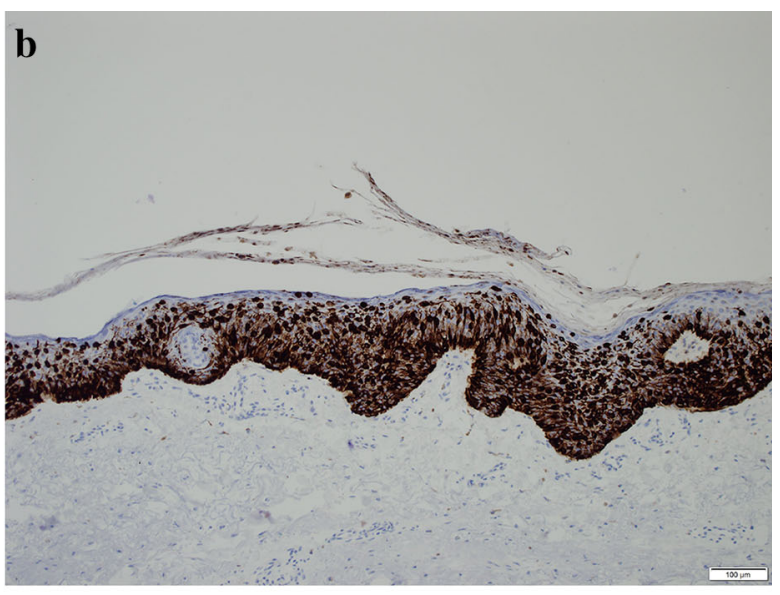

There is no evidence of dermal invasion. b Immunoperoxidase stain for Melan-A highlights the melanocytic cells of the melanoma in situ. c Tangential biopsy of the left forearm post-treatment with imiquimod, 5-fluorouracil and tretinoin revealed only a dermal cicatrix

with instructions. Three months after the conclusion of his treatment and with easing of the pandemic restrictions, the patient followed up with his dermatologist and had two tangential whole-lesional site biopsies, both of which demonstrated dermal cicatrix and absence of MIS (Fig. 2c).

\section{DISCUSSION}

Although the standard treatment of MIS is a surgical modality, the pandemic period warranted a non-surgical approach. Many medical organizations also recommended the delay of 
skin cancer treatments during the pandemic, but some reports expressed dissenting opinions about delaying the evaluation and treatment of pigmented lesions [1, 15-17]. Studies have demonstrated the efficacy of topical treatments of melanoma or MIS with imiquimod as monotherapy [8-10, 14, 18-24], imiquimod and 5-fluorouracil as dual therapy [11], and imiquimod and tazarotene as dual therapy [12]. Although tazarotene has been shown to have efficacy against MIS while tretinoin has not $[13,25]$, we chose a triple combination with tretinoin (imiquimod, 5-fluorouracil, tretinoin) over the combination with tazarotene as treatment. In the authors' experience, patients with skin type I and a history of extensive sun damage background display unacceptable amounts of inflammation with a tazarotene combination therapy. Other patients with MIS who have darker skin types and less past sun exposure may do well with the latter combination.

Imiquimod is thought to act through the innate immune system as Toll-like receptors 7 and 8 agonists that can inhibit the proliferation of human melanocytes [26] and induce proapoptotic effects in melanoma cells [27, 28]. Additionally, through their distinct mechanisms of action, 5-fluorouracil (anti-metabolite) [11] and imiquimod (immune system enhancement) $[9,10]$ are thought to provide synergistic antitumor effects. Imiquimod stimulates the production of numerous inflammatory cytokines that upregulate the enzyme thymidine phosphorylase, which is accountable for converting 5-fluorouracil to its functional end product $[29,30]$. This synergism, combined with the proposed penetration enhancement with retinoids [12] and chemoprevention offered by tretinoin, could result in the use of less medication, fewer side effects, improved clearance, and shorter duration of treatment.

Since the amount of inflammation is an essential measure of the treatment's efficacy and a potential cause for discontinuation of therapy, the use of the store-and-forward app is an indispensable tool for monitoring and guiding the patient's treatment course. The app determines whether the patient, based on screening questions, is eligible to start the treatment. The app will ask questions that screen for illness, fever, pregnancy status, among others. Although the patient can submit medical journal visits with template model photography at any time during their subscription service (Fig. 1b), the patient is prompted by email once a week to submit a journal visit. The app then allows for the transformation of the images and sequentially displays images for the physician to view in an efficient manner (Fig. 1b). The symptoms are aggregated and graded with a proprietary numerical score that allows the physician to gauge the severity of symptoms. Based on the symptoms, the patient, prompted through the app, will continue treatments, cease or delay treatments, and/or use steroids to abate symptoms. Since some symptoms of COVD-19 can mimic the flu-like syndrome of imiquimod [31], app-based decision-making is an important part of the treatment. In general, imiquimod mostly induces mild to moderate symptoms, but severe flu-like syndromes can occur [32]. Previous studies using the same treatment regimen (imiquimod, 5-fluorouracil, and tretinoin) on individual keratinocyte carcinomas also demonstrated that treatments induce mostly mild to moderate symptoms [33, 34].

There has been extensive controversy in the delay of the treatment of melanomas during the pandemic. The NCCN recommends that during a pandemic, treatments for $\mathrm{T} 0-\mathrm{T} 1$ cutaneous melanomas can be delayed for up to 3 months if there is no obvious residual lesion present after histological biopsy. The NCCN also recommends delaying treatments of cutaneous melanomas in patients with greater than or equal to T2 disease for up to 3 months if biopsy margins are negative [1]. Others have differing opinions about diagnosing and treating melanomas in that they would perform wider excisions but delay sentinel node biopsies and only perform procedures in an outpatient setting, which would limit the risks for in-patient hospitalization [35]. The European Society for Medical Oncology suggests that all patients being treated for advanced melanomas obtain swabbings for SARS-CoV-2 but continue with surgery, radiation treatments, chemotherapy, or immunotherapy [4]. Some dermatology 
departments have stressed that delays in detecting and treating melanomas can lead to increased health costs, morbidity, and mortality [3]. The British Association of Plastic Reconstructive and Aesthetic Surgeons recommends that melanoma treatments should not notably change from current established high standards [36], while some dermatologists have called for an entire cessation of non-emergent dermatological care during the pandemic [2].

Although we are recommending a treatment paradigm for melanoma using teledermatology that follows guidelines and demonstrates costefficiency and ethical compliance, the diagnosis of melanoma with teledermatology may be difficult and has not been conclusively proven $[16,37,38]$. The diagnosis of melanomas de novo may be enhanced dramatically with teledermoscopy [39], but patients may not be able to obtain such equipment during a pandemic. The ability to transform images and utilize template model photography may be of great benefit in telemedicine, but patients contribute to a poor diagnosis by being untrained in medical photography. Therefore, telemedicine may be better suited for following and treating a cutaneous melanoma rather than diagnosing one.

Our patient, who was relatively healthy with a only a few risk factors (age and asthma) for severe symptoms with COVID-19, was an ideal candidate for this treatment protocol. This treatment regimen may be even more warranted for patients with multiple risk factors for developing dangerous symptoms with COVID19 , such as significantly advanced age, lung problems, weakened immune system, cancers, chronic kidney or liver disease, certain blood disorders, heart disease, diabetes, and obesity [40-44].

\section{CONCLUSIONS}

This treatment paradigm with store-and-forward technology complies with pandemic guidelines, decreases risk of COVID-19 exposure to patients and medical staff, demonstrates costeffectiveness, and provides accurate details about inflammation. Moreover, this is the first reported case of MIS being treated with telehealth, a lack of in-person visits, and topical treatments. It is an example of how to manage MIS remotely during a pandemic crisis until an in-person visit is possible.

\section{ACKNOWLEDGEMENTS}

We thank the participant of the study.

Funding. No funding or sponsorship was received for this study or publication of this article. The rapid service fee was funded by the authors.

Authorship. All named authors meet the International Committee of Medical Journal Editors (ICMJE) criteria for authorship for this article, take responsibility for the integrity of the work as a whole, and have given their approval for this version to be published.

Disclosures. William Nahm, Eran Gwillim, Evangelos Badiavas, Anna Nichols, Robert Kirsner, Laurence Boggeln have nothing to disclose. John Shen serves as a consultant for DirectResponse Medicine.

Compliance with Ethics Guidelines. Informed consent was obtained from the patient for publication of the article, including clinical photographs.

Data availability. Data sharing does not apply to this article, as no data sets were generated or analyzed during the current study.

Open Access. This article is licensed under a Creative Commons Attribution-NonCommercial 4.0 International License, which permits any non-commercial use, sharing, adaptation, distribution and reproduction in any medium or format, as long as you give appropriate credit to the original author(s) and the source, provide a link to the Creative Commons licence, and indicate if changes were made. The images or other third party material in this article are included in the article's Creative Commons licence, unless indicated otherwise in a credit 
line to the material. If material is not included in the article's Creative Commons licence and your intended use is not permitted by statutory regulation or exceeds the permitted use, you will need to obtain permission directly from the copyright holder. To view a copy of this licence, visit http://creativecommons.org/licenses/by$\mathrm{nc} / 4.0 /$.

\section{REFERENCES}

1. Baumann BC, MacArthur KM, Brewer JD, et al. Management of primary skin cancer during a pandemic: multidisciplinary recommendations. Cancer. 2020;126(17):3900-06. https://doi.org/10. $1002 /$ cncr.32969.

2. Kwatra SG, Sweren RJ, Grossberg AL. Dermatology practices as vectors for COVID-19 transmission: a call for immediate cessation of nonemergent dermatology visits. J Am Acad Dermatol. 2020;82(5): e179-80.

3. Villani A, Fabbrocini G, Costa C, Scalvenzi M. Melanoma screening days during the coronavirus disease 2019 (COVID-19) pandemic: strategies to adopt. Dermatol Ther. 2020;10(4):525-7.

4. Conforti C, Giuffrida R, Di Meo N, Zalaudek I. Management of advanced melanoma in the COVID-19 era. Dermatol Ther. 2020;33(4):e13444.

5. Pathoulas JT, Stoff BK, Lee KC, Farah RS. Ethical outpatient dermatology care during the coronavirus (COVID-19) pandemic. J Am Acad Dermatol. 2020;82(5):1272-3.

6. Gorrepati PL, Smith GP. Analysis of availability, types, and implementation of teledermatology services during COVID-19. J Am Acad Dermatol. 2020;83(3):958-9.

7. Elsner P. Teledermatology in the times of COVID19-a systematic review. JDDG 2020;18(8):841-5.

8. Shi VY, Tran K, Patel F, et al. 100\% Complete response rate in patients with cutaneous metastatic melanoma treated with intralesional interleukin (IL)-2, imiquimod, and topical retinoid combination therapy: results of a case series. J Am Acad Dermatol. 2015;73(4):645-54.

9. Fan Q, Cohen S, John B, Riker AI. Melanoma in situ treated with topical imiquimod for management of persistently positive margins: a review of treatment methods. Ochsner J. 2015;15(4):443-7.
10. Wolf IH, Smolle J, Binder B, et al. Topical imiquimod in the treatment of metastatic melanoma to skin. Arch Dermatol. 2003;139(3):273-6.

11. Florin V, Desmedt E, Vercambre-Darras S, Mortier L. Topical treatment of cutaneous metastases of malignant melanoma using combined imiquimod and 5-fluorouracil. Invest New Drugs. 2012;30(4): 1641-5.

12. Menzies S, Mc Menamin M, Barry R. Lentigo maligna successfully treated with combination therapy of topical tazarotene and imiquimod. Clin Exp Dermatol. 2017;42(4):468-70.

13. Meyskens FL, Edwards L, Levine NS. Role of topical tretinoin in melanoma and dysplastic nevi. J Am Acad Dermatol. 1986;15(4):822-5.

14. Astorino $\mathrm{S}$, Astorre $\mathrm{P}$, Pasquini $\mathrm{P}$, et al. Imiquimod $5 \%$ cream in occlusion, for the treatment of lentigo maligna: a new scheme of short cycles and the need for clinical trials. Dermatol Ther. 2018;32:e12757.

15. Gomolin T, Cline A, Handler MZ. The danger of neglecting melanoma during the COVID-19 pandemic. J Dermatolog Treat. 2020;31(5):444-5.

16. Chuchu N, Dinnes J, Takwoingi Y, et al. Teledermatology for diagnosing skin cancer in adults. Cochrane Database Syst Rev. 2018;12(12): Cd013193.

17. Conic RZ, Cabrera CI, Khorana AA, Gastman BR. Determination of the impact of melanoma surgical timing on survival using the national cancer database. J Am Acad Dermatol. 2018;78(1):40-6.e7.

18. Verga E, Chohan B, Verdolini R. Malignant melanoma treated with topical imiquimod: a bespoke treatment that spared the amputation. Case Rep Dermatol. 2019;11:1-6.

19. Read T, Webber S, Thomas J, et al. Protocol for the TIDAL melanoma study: topical imiquimod or diphenylcyclopropenone for the management of cutaneous in-transit melanoma metastases-a phase II, single centre, randomised, pilot study. BMJ Open. 2017;7(10):e016816.

20. Ahmed I, Berth-Jones J. Imiquimod: a novel treatment for lentigo maligna. $\mathrm{Br} \mathrm{J}$ Dermatol. 2000;143(4):843-5.

21. Missall TA, Hurley MY, Fosko SW. Lentiginous melanoma in situ treatment with topical imiquimod: need for individualized regimens. Arch Dermatol. 2010;146(11):1309-10.

22. Mora AN, Karia PS, Nguyen BM. A quantitative systematic review of the efficacy of imiquimod monotherapy for lentigo maligna and an analysis of 
factors that affect tumor clearance. J Am Acad Dermatol. 2015;73(2):205-12.

23. Miller AK, Dusing R, Meggison A, Aires D. Regression of internal melanoma metastases following application of topical imiquimod to overlying skin. J Drugs Dermatol. 2011;10(3):302-5.

24. Tio D, van der Woude J, Prinsen CAC, et al. A systematic review on the role of imiquimod in lentigo maligna and lentigo maligna melanoma: need for standardization of treatment schedule and outcome measures. J Eur Acad Dermatol Venereol. 2017;31(4):616-24.

25. Rivers JK, McCarthy WH. No effect of topical tretinoin on lentigo maligna. Arch Dermatol. 1991;127(1):129.

26. Kang HY, Park TJ, Jin SH. Imiquimod, a Toll-like receptor 7 agonist, inhibits melanogenesis and proliferation of human melanocytes. J Invest Dermatol. 2009;129(1):243-6.

27. Schön MP, Wienrich BG, Drewniok C, et al. Death receptor-independent apoptosis in malignant melanoma induced by the small-molecule immune response modifier imiquimod. J Invest Dermatol. 2004;122(5):1266-76.

28. Schön M, Bong AB, Drewniok C, et al. Tumor-selective induction of apoptosis and the small-molecule immune response modifier imiquimod. J Natl Cancer Inst. 2003;95(15):1138-49.

29. Modi G, Jacobs AA, Orengo IF, McClung A, Rosen T. Combination therapy with imiquimod, 5-fluorouracil, and tazarotene in the treatment of extensive radiation-induced Bowen's disease of the hands. Dermatol Surg. 2010;36(5):694-700.

30. Ondo AL, Mings SM, Pestak RM, Shanler SD. Topical combination therapy for cutaneous squamous cell carcinoma in situ with 5-fluorouracil cream and imiquimod cream in patients who have failed topical monotherapy. J Am Acad Dermatol. 2006;55(6):1092-4.

31. Ali I, Alharbi OML. COVID-19: disease, management, treatment, and social impact. Sci Total Environ. 2020;728:138861.

32. Scarfì F, Patrizi A, Veronesi G, et al. The role of topical imiquimod in melanoma cutaneous metastases: a critical review of the literature. Dermatol Ther. 2020;1:e14165.

33. Nahm WJ, Shen J, Zito PM, et al. A non-surgical and cost-effective treatment approach employing topical imiquimod, 5-fluorouracil, and tretinoin for primary NMSCs. J Drugs Dermatol. 2021. https:// doi.org/10.36849/JDD.2021.5427.

34. Nahm WJ, Badiavas EV, Kirsner RS, et al. Treating keratinocyte carcinomas with a combination of imiquimod, 5-fluorouracil, and tretinoin using store-and-forward telemedicine in the age of coronavirus disease 2019 to promote social distancing. JAAD Case Rep. 2020;6(9):931-4.

35. Nahm SH, Rembielak A, Peach H, Lorigan PC, Contributing C. Consensus guidelines for the management of melanoma during the COVID-19 pandemic: surgery, systemic anti-cancer therapy, radiotherapy and follow-up. Clin Oncol Royal College Radiol Great Br. 2020;1:936-555.

36. British Association of Plastic Reconstructive and Aesthetic Surgeons. Advice for managing melanoma patients during coronavirus pandemic. 2020. http://www.bapras.org.uk/docs/default-source/ covid-19-docs/corona-virus-melanoma-finalversion-2.pdf?sfvrsn=2. Accessed Dec 2020.

37. Warshaw EM, Lederle FA, Grill JP, et al. Accuracy of teledermatology for pigmented neoplasms. J Am Acad Dermatol. 2009;61(5):753-65.

38. Warshaw EM, Hillman YJ, Greer NL, et al. Teledermatology for diagnosis and management of skin conditions: a systematic review. J Am Acad Dermatol. 2011;64(4):759-72.

39. Bruce AF, Mallow JA, Theeke LA. The use of teledermoscopy in the accurate identification of cancerous skin lesions in the adult population: a systematic review. J Telemed Telecare. 2018;24(2): 75-83.

40. Liu D, Cui P, Zeng S, et al. Risk factors for developing into critical COVID-19 patients in Wuhan, China: a multicenter, retrospective, cohort study. EClinicalMedicine. 2020;1:25.

41. Jordan RE, Adab P, Cheng KK. Covid-19: risk factors for severe disease and death. BMJ. 2020;368:m1198.

42. Gansevoort RT, Hilbrands LB. CKD is a key risk factor for COVID-19 mortality. Nat Rev Nephrol. 2020;2:3-15.

43. Docherty AB, Harrison EM, Green CA, et al. Features of 20133 UK patients in hospital with covid19 using the ISARIC who clinical characterisation protocol: prospective observational Cohort study. BMJ. 2020;369:m1985.

44. Robilotti EV, Babady NE, Mead PA, et al. Determinants of COVID-19 disease severity in patients with cancer. Nat Med. 2020;26(8):1218-23. 